\title{
Abrasividad y su influencia en el rendimiento de una excavación mecanizada
}

\section{Abrasiveness and its influence on the performance in continuous driving}

Fecha de entrega: 23 de enero 2013

Fecha de aceptación: 9 de abril 2013

\section{Claudia González, Marcos Arroyo y Antonio Gens}

Departamento de Ingeniería del Terreno, Cartográfica y Geofísica, Universidad Politécnica de Cataluña, Campus Diagonal Nord, Edificio D2.C, Jordi Girona, 1-3, 08034 Barcelona, España, clamaria2009@hotmail.com, marcos.arroyo@upc.edu, antonio.gens@upc.edu

El empleo de tuneladoras TBM (Tunnel Boring Machine) es cada vez más frecuente aunque su optimización requiere la evaluación precisa de varios riesgos geotécnicos entre ellos, el efecto de la abrasividad del suelo en el coste y planificación de un proyecto dado. El desgaste de las herramientas es más dificil de calcular que el resto de parámetros de la maquinaria, debido a la complejidad de la interacción entre herramienta de corte y terreno. En este trabajo se usan datos de un caso real de túnel en terrenos mixtos suelo-roca para examinar el resultado de varias metodologías de predicción de la penetración de TBM. También se examinan algunas predicciones de desgaste y se describe y aplica un nuevo método para la evaluación de la abrasividad de geomateriales que puede aplicarse tanto a materiales sueltos como a rocas.

Palabras clave: desgaste de herramientas de corte, abrasividad, rendimiento de tuneladoras
The use of Tunnel Boring Machines TBM is becoming more and more frequent although its optimization requires the accurate evaluation of various geotechnical risks, among them, the effect of soil abrasiveness in the cost and planning of a given project. Tool wear is more difficult to calculate than the rest of the machine parameters, due to the complexity of the interaction between cutting tools and ground. In this piece of work data is used from a tunnel real case in mixed ground rock-soil to examine the result of various methodologies for the prediction of TBM penetration. Also some wear predictions are examined and a new method for the evaluation of the geomaterials abrasiveness is described and applied which can be applied both to loose materials and rock.

Keywords: cutting tool wear, abrasiveness, TBM performance

\section{Introducción}

El rendimiento de una excavación mecanizada depende de dos conceptos fundamentales, por una parte del grado de penetración $\operatorname{Pr}$ y por otra del grado de avance $A r$. El grado de penetración $P r$ resulta de la relación entre la longitud excavada y el tiempo de operación durante un tramo de excavación continua, es decir, sin paradas. El grado de avance $\mathrm{Ar}$ es el cociente entre la distancia real perforada y fortificada y el tiempo total que se consume para hacerlo, incluyendo el tiempo de paradas. De ambas definiciones se deduce que $A r$ es considerablemente inferior a $\operatorname{Pr}$ y la relación entre ellos nos da un Coeficiente de Utilización U indicativo del desempeño de la máquina tuneladora en una determinada perforación.

$\mathrm{U}=\frac{A r}{P r}$

Por ejemplo, en macizos rocosos de muy baja calidad, lo más probable es que $\mathrm{Pr}$ sea alto mientras que $\mathrm{Ar}$ sea muy bajo, lo que dará un coeficiente de utilización menor del habitual. Sapigni et al. (2002) comentan que un valor estándar de U está entre un 30 y un 50\%. 
Los tiempos de parada durante la perforación pueden clasificarse en los debidos a mantenimiento programado y los debidos a incidentes imprevistos. Si bien estos últimos pueden resultar definitivos a la hora de valorar el éxito de una perforación, por definición no se consideran en proyecto. En cambio, y precisamente para reducir en la medida de lo posible, el ámbito de la sorpresa/ incertidumbre, el mantenimiento de la máquina sí debe ser objeto de proyecto (Burger, 2006).

Uno de los aspectos del mantenimiento más exigentes desde el punto de vista operativo es el de los útiles de corte, pues su revisión y eventual sustitución siempre exige una parada de la máquina y, puesto que a menudo es necesario acceder al frente, ésta es con frecuencia una operación lenta y difícil (Frenzel et al., 2008). Es así deseable programar con la mayor precisión posible las operaciones de mantenimiento de la cabeza de corte. Para efectuar tal programación son necesarios dos aspectos: la identificación de un valor límite de desgaste de las herramientas y un medio que permita relacionar el desgaste de la herramienta con el recorrido previsto para la misma, es decir, un modelo que permita estimar el desgaste de cada herramienta debido a la operación.

Son bastantes los modelos y procedimientos hoy disponibles en la literatura técnica para estimar la penetración y el desgaste de las herramientas (Bilgin y Balci, 2005; Burger, 2006; Nielsen et al., 2006a,b; Kahraman et al., 2003). Aún así, hay todavía un grado de incertidumbre importante respecto a las capacidades de predicción cuantitativa de estos procedimientos (Plinninger y Restner, 2008). Por otra parte, es notable que buena parte de estos modelos se han desarrollado y/o calibrado únicamente para trabajos de perforación en roca (Ozdemir y Wang, 1979; Ozdemir, 2003). Sin embargo, son cada vez más numerosas las ocasiones en que una máquina debe perforar un túnel donde se encontrará rocas y suelos, frecuentemente en la misma sección. Aunque en términos generales la perforación en suelos (materiales sueltos) es más fácil desde este punto de vista, no ocurre siempre así y, de hecho, hay algunos materiales sueltos, como por ejemplo gravas y bolos, que pueden causar daños muy considerables a la maquinaria (Nielsen et al., 2006a). Es por ello deseable investigar procedimientos que permitan su aplicación también en estas situaciones (Festl, 2006).

En este artículo se presentan algunos resultados de un programa de investigación destinado a responder, siquiera parcialmente, a estas cuestiones. Se emplean datos resultantes de varios proyectos de túneles recientemente finalizados en el área de Barcelona para relacionar sistemáticamente el rendimiento y desgaste observados con las propiedades de la maquinaria, de su operación y del terreno. Los resultados que se presentan aquí son de dos tipos: por un lado se examina la capacidad de distintos métodos disponibles en la literatura para reproducir la penetración observada en un tramo de túnel. Por otro lado se describe e ilustra con resultados una metodología experimental (ensayo LCPC) para la clasificación de la abrasividad de materiales que tiene aplicación para suelos y rocas (Käsling y Thuro, 2010; Thuro et al., 2006). Antes de presentar tales resultados se describe brevemente el caso de estudio.

\section{DESCRIPCIÓN DEL CASO}

\section{Proyecto L9 Tramo Can Zam - Macropou}

La red de metro de la ciudad de Barcelona se ha desarrollado históricamente sobre un concepto de distribución radial. Para complementar esta red, se introduce una línea periférica concéntrica, esta nueva línea es la Línea 9 (L9) del metro de Barcelona que, con unos $50 \mathrm{~km}$, interconecta con todas las líneas tanto de metro como de cercanías existentes e importantes instalaciones públicas como el Aeropuerto, la Fira de Barcelona, la Ciudad de la Justicia, el Camp Nou, etc.

El tramo 4A de L9 tiene una longitud aproximada de 4,5 $\mathrm{km}$ y el túnel se extiende entre Bon Pastor y Can Zam, entre el P.K. 1+355 y el P.K. 5+633. La tuneladora entró en el extremo de Can Zam, el pozo de salida se situó en Can Peixauet entre el P.K. 1+000 y P.K. 1+355. La obra comenzó en junio de 2003 y el tramo correspondiente entró en servicio el 13 de diciembre de 2009.

\section{Máquina TBM}

Para el tramo 4A se empleó una TBM escudada de tipo Dual para roca con posibilidad de trabajar en modo cerrado EPB, las características de la máquina se resumen en la Tabla 1. 
Tabla 1: Características de la máquina tuneladora

\begin{tabular}{ll}
\hline Características de la máquina & $\begin{array}{l}\text { UTE L9, tipo } \\
\text { convertible }\end{array}$ \\
\hline Diámetro nominal, mm & 11950 \\
Fabricante: franco alemana & NFMWIRTH \\
Segmento de aro (Diám.Int. - espesor), mm & $10900-350$ \\
Longitud del escudo, mm & 12590 \\
RPM: & $0-3,7$ \\
Motor de la rueda de corte: & Eléctrico \\
Potencia instalada, kW & 7335 \\
Par nominal de la cabeza de corte, kNm & 28930 \\
Torque excep. de la cabeza de corte, $\mathrm{kNm}$ & 37000 \\
Cilindros de empuje: & 30 \\
Empuje total nominal, kN & 90000 \\
Empuje total excepcional, kN & 110000 \\
Presión nominal y máx. de EPB, MPa & 0,3 \\
Radio de giro mínimo, $\mathrm{m}$ & 270 \\
\hline
\end{tabular}

\section{Descripción Geológico/Geotécnica}

Las grandes unidades geológicas y geotécnicas interceptadas por la TBM en este tramo son las siguientes: rocas ígneas paleozoicas, unidades metasedimentáreas paleozoicas, unidades mesozoicas triásicas y unidades terciarias neógenos (Tabla 3, página siguiente). En la Tabla 2 se sintetizan los parámetros que caracterizan a los materiales interceptados por la perforación y sus propiedades relacionadas con la abrasividad.

Tabla 2: Características geomecánicas de los materiales

\begin{tabular}{lllllll}
\hline $\begin{array}{l}\text { Caract. } \\
\text { medias }\end{array}$ & $\begin{array}{l}\text { Gr1 } \\
\text { (I,II) }\end{array}$ & $\begin{array}{l}\text { Gr1,2 } \\
\text { (III) }\end{array}$ & $\begin{array}{l}\text { Gr2 } \\
\text { (IV,V) }\end{array}$ & Pf & M & Co \\
\hline RMR & 52 & 45 & - & 54 & - & 45 \\
$\begin{array}{l}\text { D. seca, } \\
\text { g/cm }\end{array}$ & 2,66 & 2,55 & 1,88 & 2,61 & 2,35 & 2,77 \\
Hum. nat., \% & 0,9 & 2,1 & 12,95 & 3,1 & 7,75 & 0,75 \\
RCS, MPa & 95 & 14,7 & 0,28 & 97 & 0,42 & 32,5 \\
E, MPa & 54000 & 7000 & 193 & 12589 & 3000 & 4742 \\
C. de & 0,26 & 0,21 & 0,35 & 0,23 & 0,35 & 0,33 \\
Poisson & & & & & & \\
$\begin{array}{l}\text { Trac.Ind., } \\
\text { MPa }\end{array}$ & 12,07 & 3,75 & - & 13,25 & - & 6,15 \\
CAI & 2,70 & - & - & - & - & 3,20 \\
$\begin{array}{l}\text { Min. Abras., } \% \\
\text { c, kg/cm }\end{array}$ & 62 & 62 & 67 & - & - & 52 \\
$\begin{array}{l}{ }^{\circ}{ }^{\circ} \\
\text { I.Schim., }\end{array}$ & 2,00 & 1,40 & 0,55 & 1,00 & 0,53 & 4,00 \\
kN/m & 5,51 & - & - & 6,88 & - & 0,27 \\
$\begin{array}{l}\text { Carga } \\
\text { puntual }\end{array}$ & - & - & - & - & - & 2,85 \\
\hline
\end{tabular}

La Figura 1 presenta un perfil esquemático en el que se identifican las unidades geotécnicas más representativas que atraviesa el túnel. Como se puede observar, existe una presencia dominante de la unidad geotécnica Gr1, aunque también se atraviesan tramos importantes de Gr2 y Bf. El Mioceno se mantiene sobre la clave del túnel, sin ser interceptado más que en una zona puntual a la altura del PK 2+040 al 1+920. Como se aprecia se trata de una perforación sobre mezcla de suelos y rocas blandas.

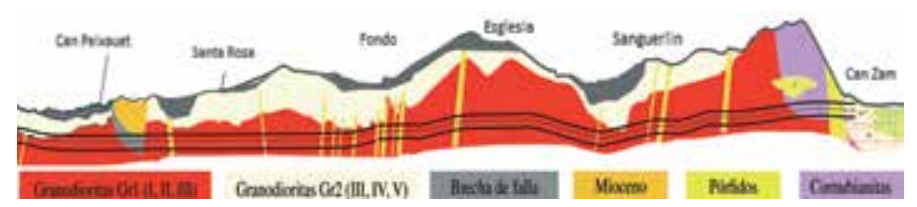

Figura 1: Esquema de perfil geotécnico túnel tramo Can Zam

\section{Predicción de rendimientos}

\section{Estimación del grado de penetración}

Son numerosas las metodologías disponibles en la literatura para estimar el valor de $P r$. Los distintos métodos o fórmulas emplean hasta tres grupos de parámetros para llegar a una predicción: parámetros de operación de la maquinaria, parámetros característicos de la roca intacta y parámetros característicos del macizo rocoso. A continuación se describe la aplicación de varias ecuaciones de predicción del grado de penetración al caso de Can Zam. En cada caso se compara la predicción con los valores observados durante la excavación. Seguimos un orden de complejidad creciente en la metodología de predicción. Obviamente, ésta dependerá directamente del número de parámetros requeridos para la predicción, $\mathrm{y}$, a igualdad de parámetros, de la mayor o menor dificultad experimental en la determinación de los mismos.

\section{Predicciones en función de parámetros de operación}

Las metodologías más sencillas de predicción de rendimiento son las que emplean únicamente parámetros de operación de la máquina. Roxbosough y Phillips (1975) asumieron que la penetración $P R_{e v}$ en $\mathrm{mm} / \mathrm{rev}$ es igual a la profundidad de indentación o corte y obtuvieron la siguiente ecuación para predecir el coeficiente de corte $C c$ :

$C_{C}=\frac{F_{r}}{F_{n}}=\sqrt{\frac{P R_{e v}}{\left(D-P R_{e v}\right)}}$ 
Tabla 3: Unidades Geológico-Geotécnicas del tramo que atraviesa el túnel.

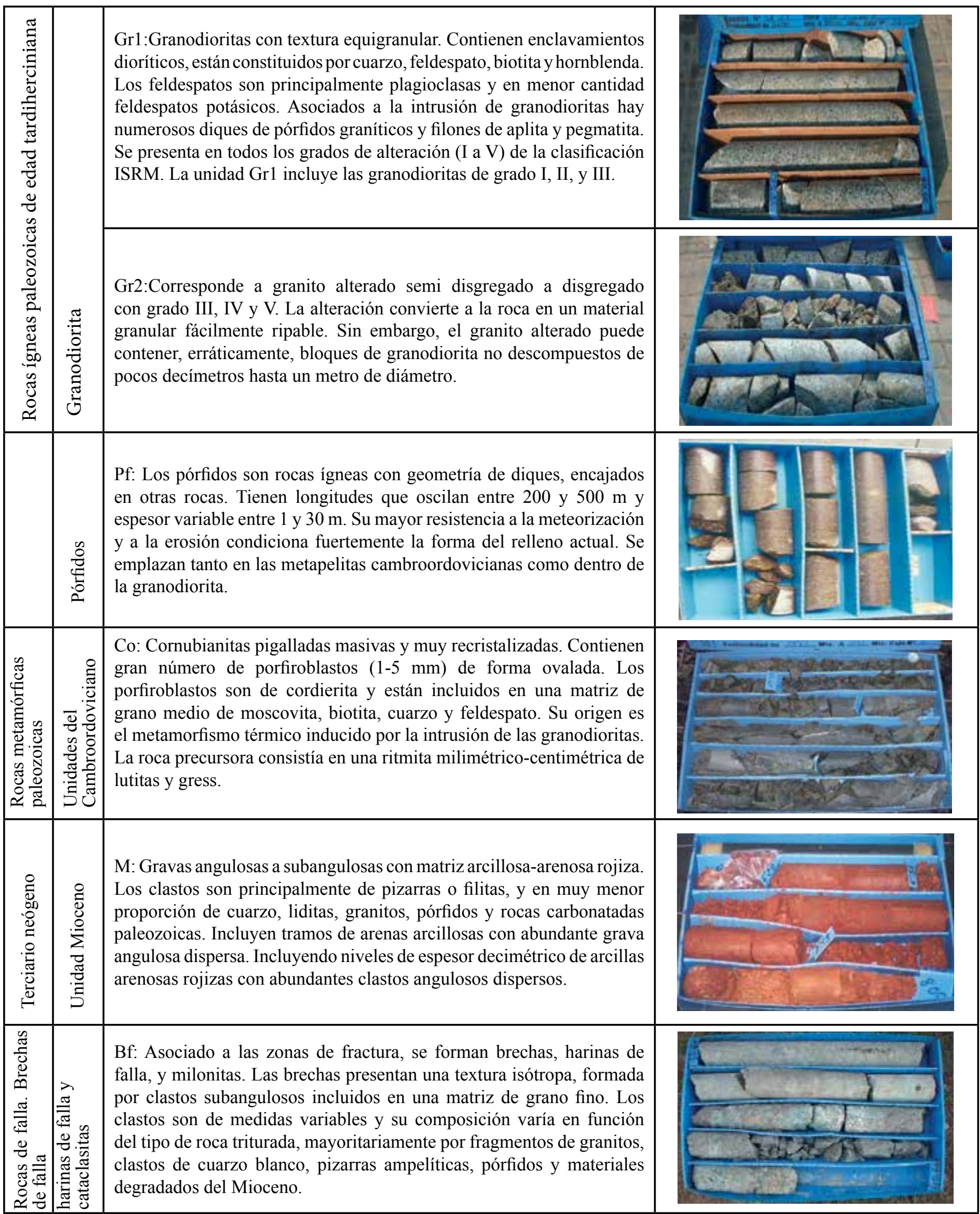


donde $F_{r}$ y $F_{n}$ son las fuerzas de rodadura y normal aplicadas al disco de corte, ambas en $\mathrm{kN}, D$ es el diámetro del disco de corte en $\mathrm{mm}, P R_{e v}$ en $\mathrm{mm} / \mathrm{rev}$.

La fuerza de rodadura $F_{r}$ se calcula como:

$$
F_{r}=\frac{P}{2 \pi n r R_{c}}
$$

donde $F_{r}$ está en $\mathrm{kN}, P$ es la potencia de la máquina, $n$ es el número de cortadores distribuidos en la cabeza de corte, $r$ es la velocidad de rotación de la cabeza de corte en rpm y $R_{c}$ es una ponderación media de la distancia de los cortadores al centro de rotación. Para el caso de estudio resulta en un valor $R_{c}$ de $54 \%$. La fuerza normal se calcula como:

$$
F_{n}=\frac{E}{f_{l} n_{c}}
$$

donde $F_{n}$ está en $\mathrm{kN}, E$ es el empuje total de la máquina en $\mathrm{kN}, f_{l}$ es un coeficiente que tiene en cuenta pérdidas friccionales, usualmente estimado en 1, 2 a 1, 3, $n_{c}$ número de cortadores. En la Figura 2 observamos que los valores pronosticados de penetración en Can Zam sobreestiman, por lo general, los observados.

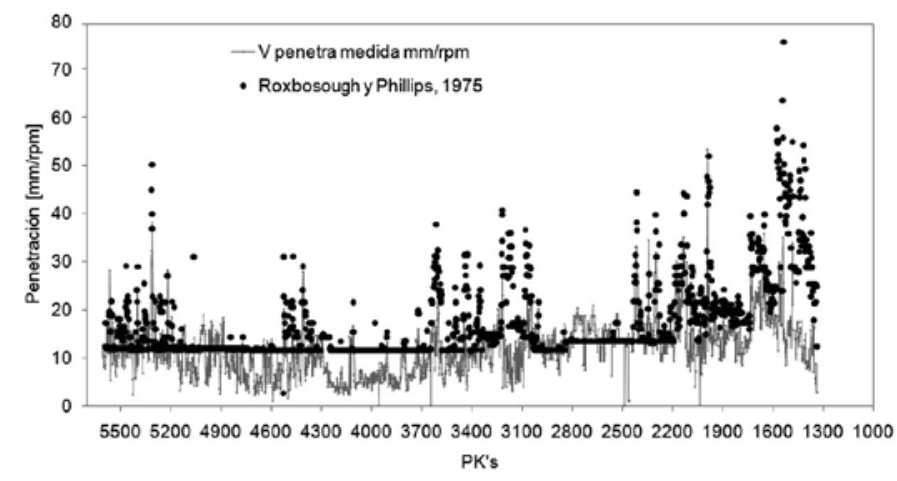

Figura 2: Valores de penetración medidos versus estimados según Roxbosough y Phillips (1975) para Can Zam

\section{Predicciones incorporando parámetros de la roca intacta}

En este apartado se consideran metodologías de predicción de rendimiento todavía relativamente sencillas, pues emplean únicamente parámetros de operación de la máquina y parámetros característicos de la roca maciza.
Así, por ejemplo, Farmer y Glossop (1980) propusieron la siguiente expresión a partir de un análisis sobre rocas sedimentarias.

$P R_{e v}=\frac{624 F_{n}}{\sigma_{t B}}$

donde $P R_{e v}$ se evalúa en $\mathrm{mm} / \mathrm{rev}$, la fuerza normal $F_{n}$ en $\mathrm{kN}$, y la resistencia a la tracción de la roca intacta $\sigma_{t B}$ en $\mathrm{kN} / \mathrm{m}^{2}$. En el caso de Can Zam se perforaron en su mayoría rocas ígneas del paleozoico y rocas metamórficas, por lo que a priori no cabría esperar muy buenos resultados. Sin embargo, la fórmula aproxima bien las medidas en varios tramos, por ejemplo entre PK2500 y PK3000, donde se atravesaron granodioritas Gr1(II) a Gr2(III) débiles y muy fracturadas. En otros tramos la discrepancia es notable, por ejemplo, entre los PK3700 y 4400, donde se predice una penetración muy superior a la observada. En ese tramo se atravesaron materiales Gr I y II duros y sin fracturas y algunas zonas de pórfidos Pf.

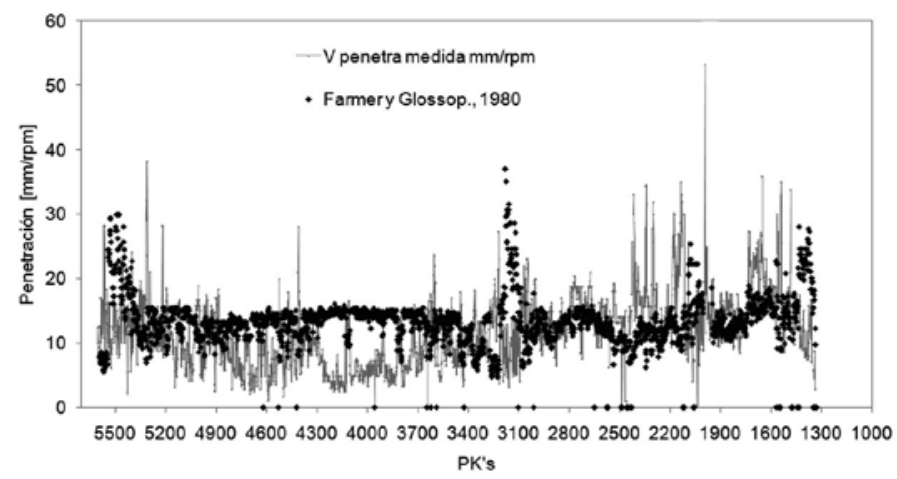

Figura 3: Valores de penetración medidos versus estimados según Farmer y Glossop (1980) para Can Zam.

Graham (1976) propuso una fórmula a partir de una base de datos de rocas duras es decir con valores de RCS entre los 140 a $200 \mathrm{MPa}$. La expresión propuesta es formalmente muy similar a la (5), si bien el parámetro que caracteriza la resistencia del terreno es distinto,

$P R_{e v}=\frac{3940 F_{n}}{R C S}$

donde $P R_{e v}$ se calcula en $\mathrm{mm} / \mathrm{rev}$, la fuerza normal $F_{n}$ en $\mathrm{kN}$ y la Resistencia a la Compresión Simple $R C S$ en MPa. En el caso real se atravesaron rocas blandas con una resistencia a la compresión simple máxima de $80 \mathrm{MPa}$ (ver Figura 4). 


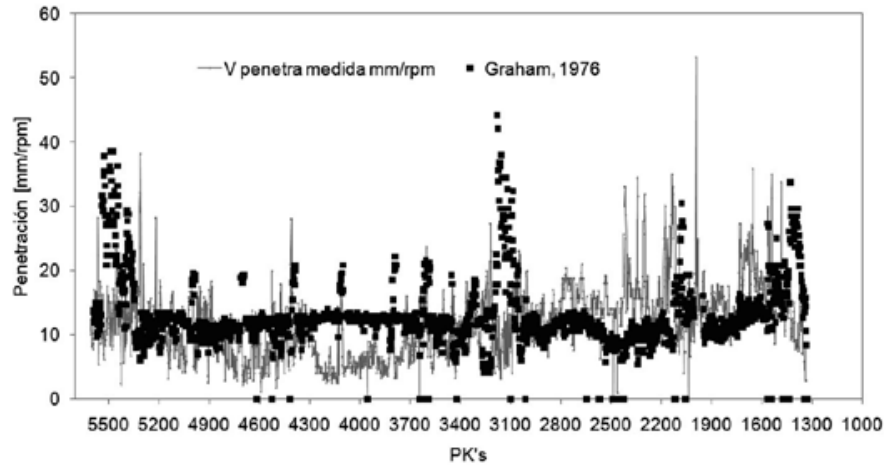

Figura 4: Valores de penetración medidos versus estimados según Graham (1976) para Can Zam

Tanto la fórmula de Farmer y Glossop como la de Graham no tienen en cuenta los parámetros del macizo rocoso y por lo tanto no son capaces de representar las variaciones por presencia o no de discontinuidades, tal como se refleja entre los tramos de PK's4800 a 4700 ó 4690 a 4630 ó 4270 a 4130 en donde el material atravesado son granitos tipo I y II sin fracturas y por lo tanto, la Penetración es mucho menor tal como se refleja en la curva de medición del caso real en la que se visualizan los valles en la curva de valores medidos. También se observa en la Figura 4 que la fórmula de Graham predice, por lo general valores ligeramente más bajos que los valores medidos. Este fenómeno se puede entender partiendo de que se llegó a esta fórmula sobre la base de datos de rocas duras es decir con valores de RCS entre los 140 a $200 \mathrm{MPa}$, mientras que las rocas que se atravesaron en el caso de Can Zam se mantuvieron siempre unos valores de $80 \mathrm{MPa}$. Por tanto la fórmula no está calibrada en el rango relevante.

Hughes (1986) propuso otra relación a partir del análisis de una base de datos en minas de carbón:

$P R_{e v}=6\left(\frac{F_{n}}{R C S}\right)^{1.2} \frac{N n}{r^{0.6}}$

donde $F_{n}$ es la fuerza normal en un disco periférico en $\mathrm{kN}$, $N$ es la velocidad de la cabeza de corte en rev/s, $n$ es el número de discos y $R C S$ está en MPa y $r$ es el radio promedio de los discos. La subestimación de la penetración medida es clara en todo el trazado como se puede observar en la Figura 5. Una vez más, esta discrepancia puede atribuirse a la falta de semejanza de los materiales presentes en la traza con los incluidos en la base de datos que subyace a la fórmula mencionada.

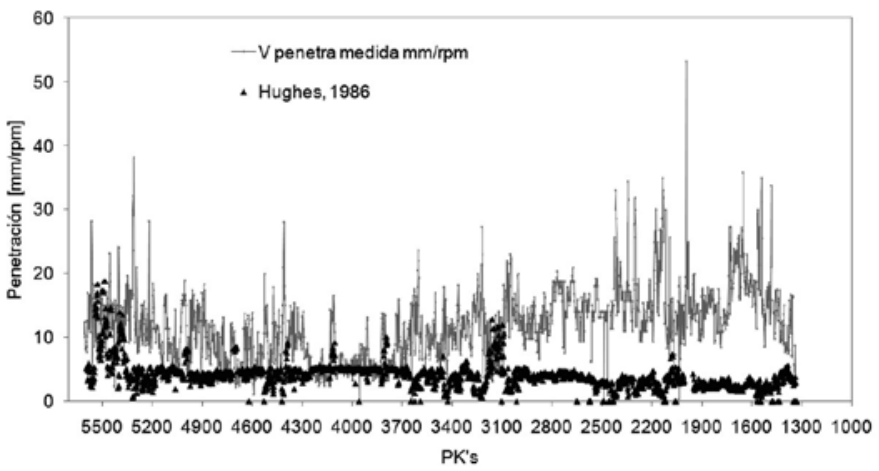

Figura 5: Valores de penetración medidos versus estimados según Hughes (1986)

\section{Predicciones incorporando parámetros del macizo rocoso}

Un grado mayor de complejidad supone la inclusión de parámetros característicos del macizo rocoso en el modelo de predicción. Por ejemplo, Gehring (1995) propuso un modelo que considera una función base y varios factores de corrección. La fórmula de base utiliza un parámetro de la roca intacta y un parámetro de operación de la máquina. Los factores de corrección más importantes $k_{i}$ tienen en cuenta la orientación de los planos de debilidad y el espaciamiento entre los planos de estratificación además del tamaño y el espaciamiento entre los cortadores.

$$
P R_{e v}=\frac{4 F_{n}}{R C S}\left(k_{1}, k_{2}, \ldots .\right)
$$

El primer factor de corrección está en función de la energía específica de fracturación $w_{f} \mathrm{y}$ dicho concepto se puede calcular según el propio Gehring como:

$$
w_{f}=0.74 \mathrm{CAI}^{1.93}
$$

Expresión que tiene en cuenta la abrasividad de la roca a través del índice CAI (Cerchar Abrasiveness Index). Luego,

$$
k_{1}=0.475 w_{f}^{-0.56}
$$

Otro factor de corrección tiene que ver con el espaciamiento $s$ entre los discos de corte con lo cual Gehring tiene en cuenta la configuración de la rueda de corte. Finalmente, el factor $k_{4}$ tiene en cuenta la influencia de trabajar con 
un aro de corte con un diámetro distinto al de 17': $k_{4}=$ $430 d i$, siendo $d i$ un diámetro de aro de corte distinto al de 17 '(430 mm).

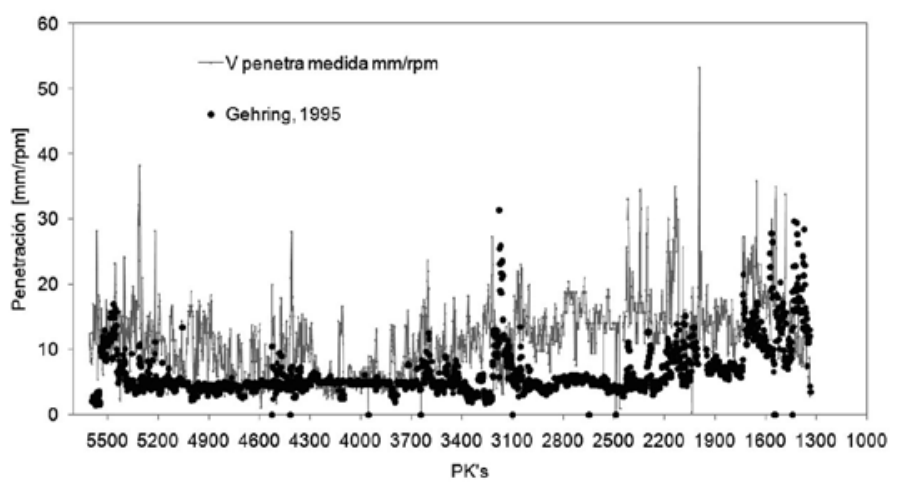

Figura 6: Valores de penetración medidos versus estimados según Gehring (1995) para Can Zam

Casinelli et al. (1982) hallaron una correlación entre $P R_{e v}$, el índice geomecánico Rock Structure Rating RSR (Bieniawski, 1989) y la Resistencia a la Compresión Simple RCS de la roca intacta:

$$
\begin{aligned}
& P R_{e v}=\sigma_{c}^{-0.437}+0.047 R S R+3.15 \\
& R S R=0.77 R M R+12.4
\end{aligned}
$$

donde $P R_{e v}$ es la penetración en $\mathrm{mm} / \mathrm{rev}$ y $\sigma_{c}$ es la resistencia a la compresión simple en MPa. Los autores plantearon su formulación sobre la base de datos de cinco túneles que suman un total de $19 \mathrm{~km}$ excavados en rocas con RCS entre 50 y $150 \mathrm{MPa}$. En la Figura 7 se han podido representar muy pocos puntos dado que no se obtuvieron valores de $R M R$ sistemáticamente a lo largo de toda la perforación.

Por su parte, Eusebio et al. (1991) propusieron modificar el modelo anterior para aplicarlo a rocas de estructura muy variable, empleando un esquema de clasificación llamado Ground Difficulty Index GDI. El GDI se obtiene a partir de los índices geotécnicos $R Q D$ y $R M R$ y la resistencia a la compresión simple in situ medida mediante martillo Schmidt. A partir de este índice se determina un factor multiplicador $f l$ que modifica el valor de penetración $P R_{e v}$ determinado según la fórmula de Casinelli et al. (1982). De la Figura 8 no parece que esta corrección mejore mucho la predicción anterior.

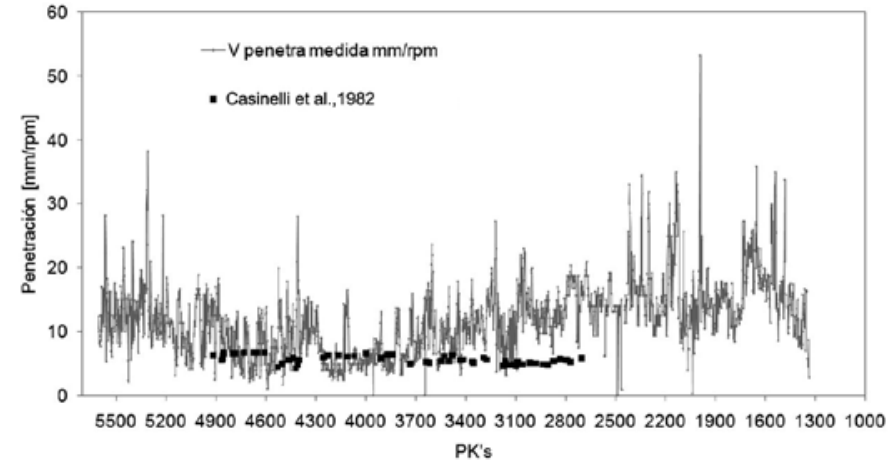

Figura 7: Valores de penetración medidos versus estimados según Casinelli et al. (1982).

Cabe señalar que estos dos métodos no tienen en cuenta ningún parámetro de operación de la máquina. Así, al aplicarlo al caso de Can Zam no pueden reproducir la variabilidad observada a lo largo del trazado. Por otra parte, la aplicación de este método resulta imposible en suelos o materiales granulares, sin una estructura que permita obtener los datos geomecánicos.

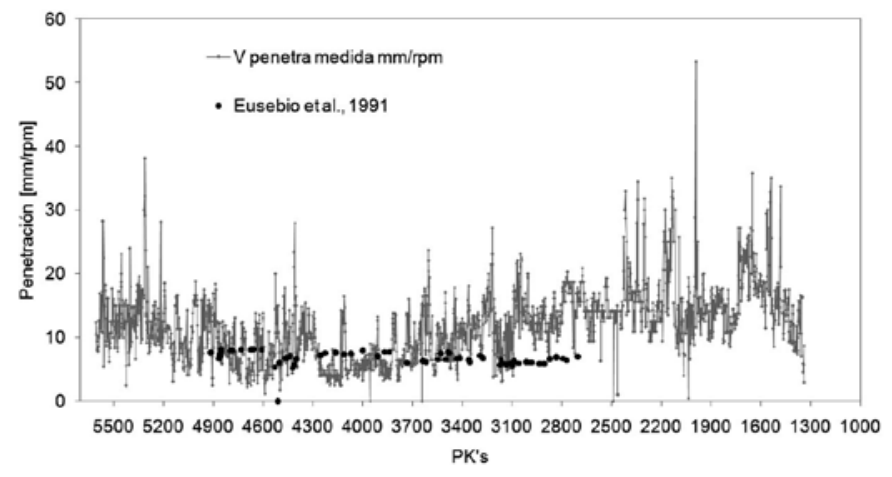

Figura 8: Valores de penetración medidos versus estimados según Eusebio et al. (1991) para Can Zam

\section{El método NTNU}

En la Universidad Noruega de Ciencia y Tecnología de Trondheim NTNU, se desarrolló ya hace 50 años una primera metodología para estimar la perforabilidad de las rocas (Lien, 1961). Esta metodología se ha actualizado repetidas veces, y es conocida como el modelo o método NTNU (Bruland, 2001; Nielsen et al., 2006b). La base empírica del método abarca más de $250 \mathrm{~km}$ de túneles (dominados por las rocas ígneas), y la predicción utiliza algunos parámetros de las tuneladoras, otros de la roca intacta y otros del macizo rocoso. 
Este método tiene un grado superior de complejidad a los anteriores, pues requiere de varios ensayos específicos, algunos de los cuales emplean una herramienta de corte real lo que implica que ya debe estar fabricada la máquina o al menos el diseño de las herramientas de corte que se van a emplear en el proyecto. Los resultados de los ensayos se correlacionan mediante la aplicación de varios ábacos. Dada la complejidad del método sólo podemos apuntar aquí algunos aspectos importantes de su aplicación a este caso.

El material se caracteriza mediante una serie de ensayos específicos: índices DRI, CLI y Ab (abrasividad). Estos pudieron medirse únicamente para una unidad geotécnica: el granito Gr1(II). Para el resto de los materiales atravesados los ensayos prescritos no pudieron llevarse a cabo porque las medidas se situaban fuera del rango de los aparatos de ensayo.

La presencia de discontinuidades (juntas y fracturas) se tiene en cuenta mediante factores de corrección que se aplican al valor de penetración básica. Estos factores dependen del espaciamiento y el ángulo que definen con el eje del túnel, como se ilustra en el ábaco de la Figura 9. En nuestro caso se estimó la separación entre discontinuidades en unos $50 \mathrm{~mm}$, a partir del valor de RQD (disponible sólo para ciertos tramos). Por otra parte, y puesto que no se dispone del dato del ángulo de inclinación relativa, se ha considerado que el factor $k_{s}$ de corrección toma un valor medio de 4 .

Los valores de penetración resultantes para la unidad geotécnica en que se obtuvo datos de laboratorio se presentan en la Figura 10. La predicción es buena, salvo en los puntos cercanos al 3800 , donde se sobrestima la penetración. En cualquier caso queda claro que, en este ejemplo, el rango de aplicación del método es restringido.

El valor de Abrasividad determinado en el procedimiento NTNU no interviene en la ecuación que determina la Penetración, sino en la estimación de la vida útil de los discos y los tiempos de parada de la máquina. En el sistema NTNU, el valor de la abrasividad permite determinar la vida útil de los discos en horas.

En este caso, y a partir de las curvas expuestas en Movinkel y Johannessen (1986) que estiman la vida de los cortadores, se ha estimado para el material GrI-II la vida útil que le correspondería a un disco en función del valor de CLI (Cutter Life Index) determinado experimentalmente y corroborado con datos previos (Gutiérrez, 2010).

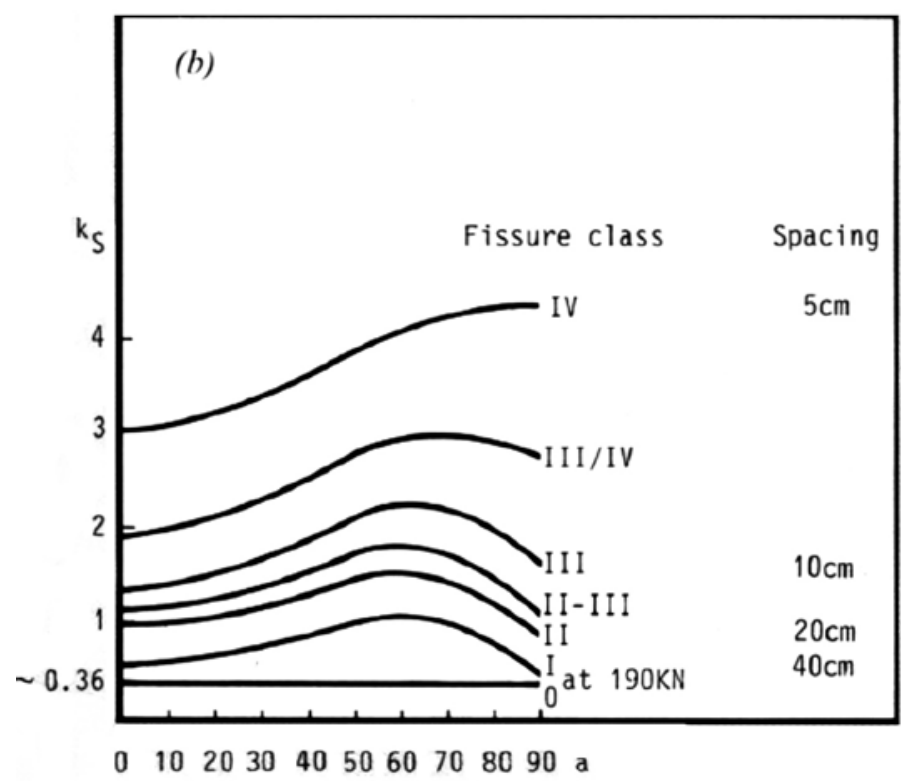

Figura 9: Abaco de $k_{s}$ en función de ángulo a y espaciamiento entre fisuras (Movinkel y Johannessen,1986)

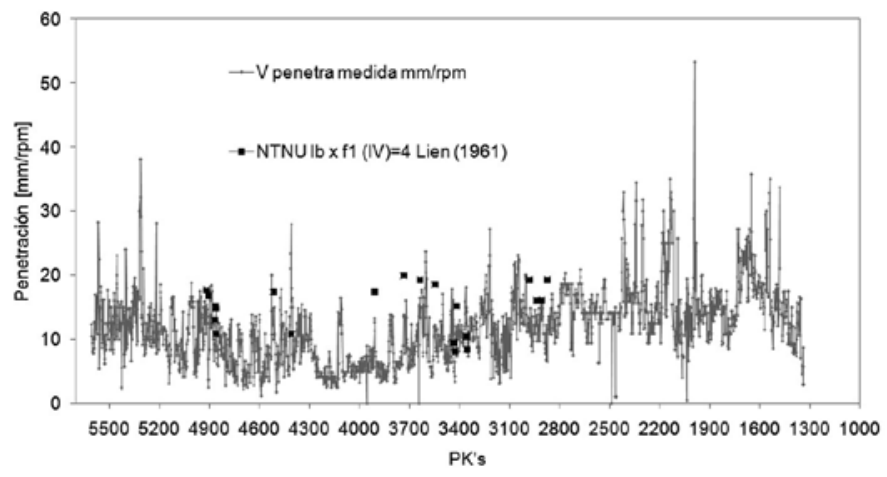

Figura 10: Valores de penetración medidos versus estimados según Bruland (2001)

Para un $C L I$ de 15 se estima unas $110 \mathrm{hr}$ de vida para un cortador promedio. Empleando los datos reales de operación de la TBM en el tramo se puede estimar el consumo, bajo el supuesto de operación continuada. Así, los valores promedio de penetración y velocidad de la cabeza de corte, son respectivamente de $17,40 \mathrm{~mm} / \mathrm{min}$ y de 3,69 rev/min. Por otra parte, los cambios de herramienta observados en la zona indican una vida media de 20 dovelas por cortador.

La Tabla 4 muestra que los valores estimados son superiores a los medidos en avance y en vida útil de cortadores en 
$m$ respectivamente. Más allá, no obstante, de lo acertado o no de esta predicción, lo que resulta evidente es que el rango de aplicación del método es muy reducido en este caso de materiales mixtos. Para poder tratar estos casos son necesarias otras herramientas, como, por ejemplo, el ensayo que se describe a continuación.

Tabla 4: Valores medidos y estimados de avance y de vida útil de un cortador medio.

\begin{tabular}{|l|c|c|}
\hline & Método noruego & Medición real \\
\hline $\begin{array}{l}\text { Avance TBM en } \\
110 \mathrm{hr}\end{array}$ & $114,84 \mathrm{~m}$ & $\begin{array}{l}20 \text { dovelas de } \\
1,8 \mathrm{~m}=36 \mathrm{~m}\end{array}$ \\
\hline $\begin{array}{l}\mathrm{km} \text { recorridos } \\
\text { por cortador }\end{array}$ & 916,7 & 476,8 \\
\hline
\end{tabular}

\section{Predicción de desgaste y grado de avance}

El desgaste de las herramientas está directamente ligado a la abrasividad de la roca/suelo. Por lo tanto la Abrasividad es una propiedad fundamental para evaluar el grado de avance $A r$ y es necesario contar con ensayos confiables que permitan determinar dicha propiedad. Los ensayos que proponen los modelos de predicción actuales apuntan sobre todo a determinar la abrasividad en rocas (Ozdemir, 2003; Cerchar, 1986; Plinninger y Restner, 2008), por ejemplo, el método Noruego (Bruland, 1998; Nielsen et al., 2006b; Movinkel y Johannessen, 1986), no pudo aplicarse a todas las unidades geotécnicas perforadas porque los ensayos que propone no admiten ser aplicados en materiales blandos ni disgregados. Para este tipo de materiales una alternativa práctica es el ensayo de broyabilité del LCPC.

\section{Ensayo de abrasividad LCPC}

Este ensayo (AFNOR, 1990) emplea un aparato que se conoce como "abrasímetro" (Figura 11). Este tiene un motor de $750 \mathrm{~W}$, que rota una paleta metálica durante 5 minutos a $4500 \mathrm{rpm}$ dentro en una vasija cilíndrica llena con las partículas del material granular. La muestra de material granular debe pesar $500 \pm 2 \mathrm{~g}$. El material granular se seca, muele y tamiza, para reducir todo el material de ensayo a la fracción entre 4 y $6,3 \mathrm{~mm}$.

La paleta metálica es un rectángulo con dimensiones 50 x 25 x $5 \mathrm{~mm}$ y está hecho de acero estandarizado con dureza Rockwell B60-75. Esta paleta de acero se cambia al finalizar cada ensayo.

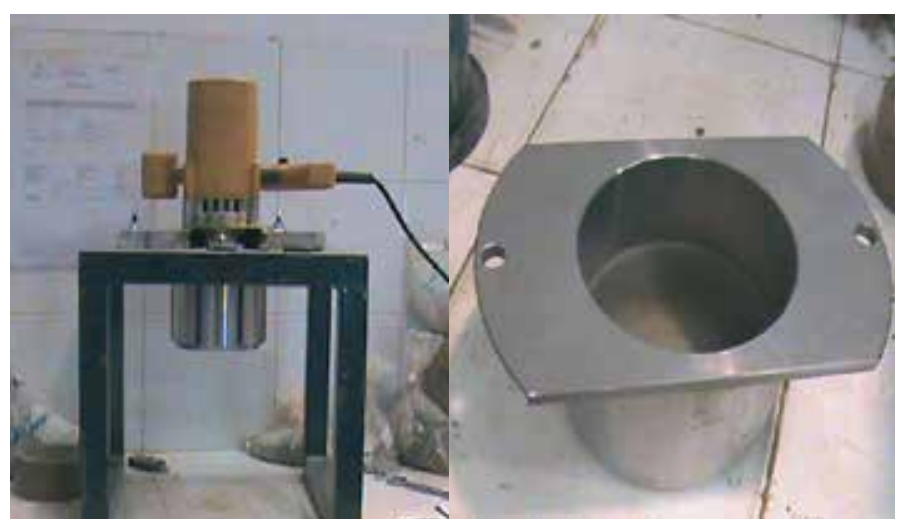

Figura 11: Máquina para ensayo LCPC

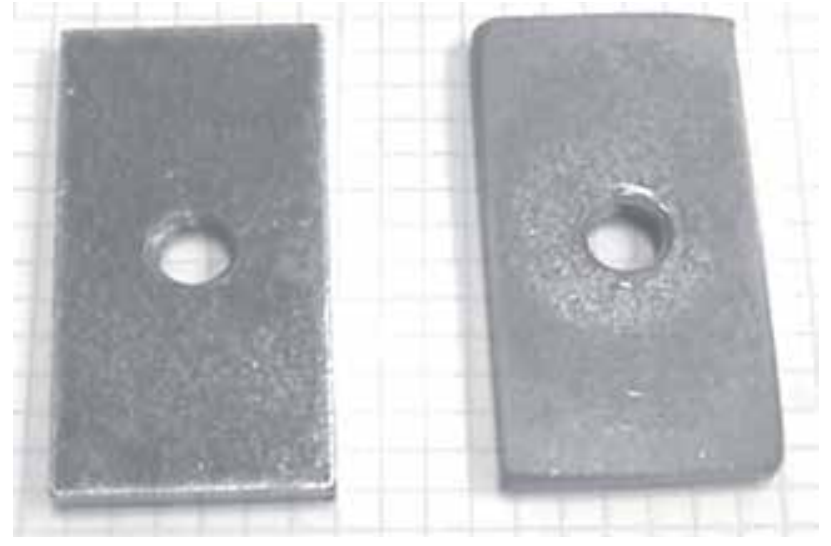

Figura 12: Paleta metálica antes y después del ensayo

La pérdida de material de la paleta metálica se debe a la resistencia del material granular sometido a la rotación en la vasija, por lo que cuanto más abrasiva sea la muestra, mayor será la pérdida de material. Para la determinación de la abrasividad, la paleta metálica ha de ser pesada antes y después del ensayo. La pérdida de peso de la paleta metálica es una medida de la abrasividad de la muestra ensayada y por lo tanto de la propiedad del material. En la Figura 12 se enseña un ejemplo de como es la paleta antes y después del ensayo y el efecto de la abrasividad en la pérdida de material y desgaste de la paleta metálica.

Se define como Coeficiente de Abrasividad LCPC Abr al cociente de la pérdida de masa de la paleta metálica por la masa del suelo (500g):

$$
A b r=\frac{m_{0}-m}{M}
$$


donde $m_{0}$ es la masa del paleta metálica antes del ensayo en $\mathrm{g}, m$ es la masa del paleta metálica después del ensayo en g y $M$ es la masa de la muestra igual a $500 \mathrm{~g}$, pero expresado en toneladas $(0,0005$ ton).

Existen dos clasificaciones para el índice de abrasividad del ensayo LCPC en la literatura. La primera clasificación fue presentada por Büchi et al. (1995), la segunda clasificación responde a un esquema desarrollado en la TUMunich (Thuro et al., 2006), ambas clasificaciones se recogen en la Tabla 5.

Tabla 5: Clasificación de Abrasividad según ensayo LCPC

\begin{tabular}{llll}
\hline \multicolumn{2}{l}{ (Büchi et al., 1995) } & \multicolumn{2}{l}{ TUM (Thuro et al., 2006) } \\
Abr, g/ton & Clasificación & Abr, g/ton & Clasificación \\
\hline$<500$ & muy baja ab & $0-50$ & no ab \\
$500-1000$ & baja ab & $50-100$ & ligeramente ab \\
$1000-1500$ & ab media & $100-250$ & poco ab \\
$1500-2000$ & alta ab & $250-500$ & abrasivo \\
$>2000$ & muy alta ab & $500-1250$ & muy ab \\
& & $1250-2000$ & extremadamente ab
\end{tabular}

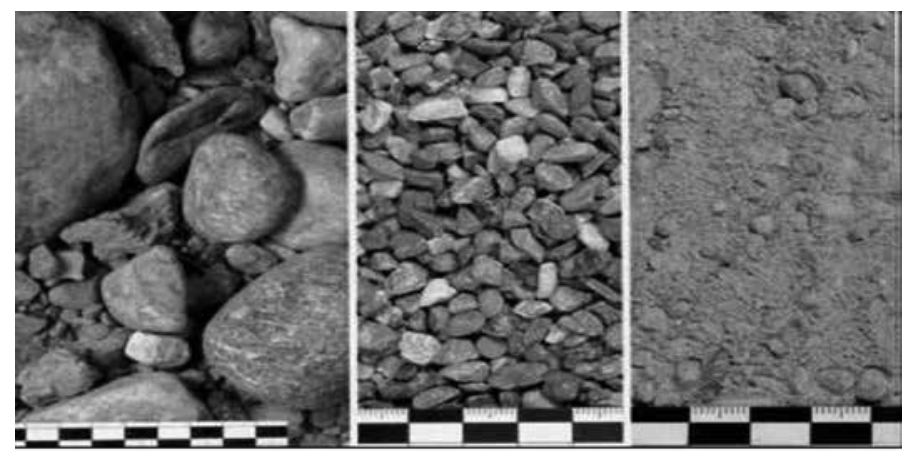

Figura 13: Muestra de grava arenosa antes de la preparación, después de la trituración y molienda a fracción entre 4 y 6,3 mm y después del ensayo de abrasividad. Una barra negra mide $1 \mathrm{~cm}$ y una pinta negra determina $1 \mathrm{~mm}$ (Thuro et al., 2006)

Como se ilustra en la Figura 13, el geomaterial ensayado también cambia notablemente durante el ensayo. Por esta razón, el ensayo también incluye un análisis granulométrico "a posteriori" para evaluar la fragmentación del material. Esta medida se traduce en un índice, llamado $L C P C$ Breakability Coeficient LBC (Thuro et al., 2006). Éste se define como la fracción por debajo de 1,6 $\mathrm{mm}$ de material de muestra tras el ensayo relativa a la misma fracción en la muestra original:
$L B C=100 M_{1,6} / M$

donde $M_{1,6}$ corresponde a la fracción de masa, de la muestra, de tamaño inferior a 1,6 $\mathrm{mm}$ después del ensayo LCPC y $M$ es la masa del material de la muestra original. El $L B C$ tiene un rango entre 0 y $100 \%$, que se ha dividido en 5 clases, como se aprecia en la Tabla 6.

Tabla 6: Clasificación según el LBC

\begin{tabular}{ll}
\hline LBC, $\%$ & Clasificación de rotura \\
\hline $0-25$ & muy baja \\
$25-50$ & baja \\
$50-75$ & media \\
$75-100$ & alta \\
$>100$ & muy alta \\
\hline
\end{tabular}

Más detalles sobre el ensayo, y en particular, sobre la sistemática de preparación de la muestra se dan en Büchi et al. (1995) y en Thuro et al. (2006).

\section{Aplicación al caso}

Con el objetivo de determinar la abrasividad para cada uno de los materiales perforados en el túnel de nuestro estudio se obtuvieron muestras de cada unidad geotécnica atravesada y se ensayaron con el método de LCPC. Los resultados se compararon con datos previos sobre abrasividad obtenidos mediante otros procedimientos habituales (Cerchar, CAI ISchimazek ISchi, Contenido Equivalente de Cuarzo CEQ, Drilling Rate Index DRI, Cutter Life Index CLI, Rock Abrasiveness Index RAI, Abrasion Value Steel AVS), véase Plinninger y Restner (2008) para una descripción de estos métodos. En general, cada método tiene su propia escala cuantitativa que no es comparable con otro método, pero a su vez resulta en una escala cualitativa que nos permite comparar sus resultados.

Allí donde se pueden comparar, los valores de abrasividad determinados por el ensayo de LCPC son cualitativamente coherentes con los obtenidos por otros métodos. Por otra parte, se observa la limitación que ofrecen ciertos ensayos en cuanto a que no son aptos para rocas blandas y materiales disgregados o granulares. El método LCPC, en cambio, es capaz de valorar todos los materiales, poniendo así de manifiesto su versatilidad. 
Tabla 7: Comparación de valores cualitativos de abrasividad entre diferentes métodos y el método de LCPC a partir de ensayos de laboratorio.

a)

\begin{tabular}{|l|c|l|c|}
\hline Material & Abr, g/ton & E. Cualit. & C.E.Q., $\%$ \\
\hline Co & 319,9 & ab media & 52,0 \\
\hline $\operatorname{Gr} 1(\mathrm{II})(50 \%)+\operatorname{Co}(50 \%)$ & 549,2 & muy ab & 57,0 \\
\hline $\operatorname{Pf}(50 \%)+\operatorname{Co}(50 \%)$ & 599,8 & muy ab & 56,5 \\
\hline $\operatorname{Gr} 1(\mathrm{II})$ & 778,4 & muy ab & 62,0 \\
\hline $\operatorname{Gr} 1(\mathrm{II})(50 \%)+\mathrm{Gr} 2(\mathrm{III})(50 \%)$ & 589,0 & muy ab & 62,0 \\
\hline $\operatorname{Bf}(80 \%)+\mathrm{M}(20 \%)$ & 59,8 & \multicolumn{2}{|l}{ no muy ab } \\
\hline
\end{tabular}

b)

\begin{tabular}{|l|c|l|l|l|}
\hline Material & Abr, g/ton & E. Cualit. & CAI & E. Cualit. \\
\hline Pf & 879,7 & muy ab & 8,00 & $\begin{array}{l}\text { fuera de } \\
\text { rango }\end{array}$ \\
\hline Co & 319,9 & ab media & 2,80 & muy alta \\
\hline $\begin{array}{l}\text { Gr1(II)(50\%)+ } \\
\text { Co(50\%) }\end{array}$ & 549,2 & muy ab & 3,45 & muy alta \\
\hline Pf(50\%)+Co(50\%) & 599,8 & muy ab & 5,40 & Extrem.Ab. \\
\hline Gr1(II) & 778,4 & muy ab & 4,10 & Extrem. Ab. \\
\hline $\begin{array}{l}\text { Gr1(II)(50\%)+ } \\
\text { Gr2(III)(50\%) }\end{array}$ & 589,0 & muy ab & 6,05 & cuarzo \\
\hline Bf (80\%)+M(20\%) & 59,8 & no muy ab & \\
\hline
\end{tabular}

c)

\begin{tabular}{|l|c|l|l|l|}
\hline Material & Abr, g/ton & E. Cualit. & DRI & E. Cualit. \\
\hline Pf & 879,7 & muy ab & 34,50 & me \\
\hline Co & 319,9 & ab media & 53,40 & media \\
\hline $\begin{array}{l}\text { Gr1(II)(50\%)+ } \\
\text { Co(50\%) }\end{array}$ & 549,2 & muy ab & 50,70 & media \\
\hline Pf(50\%)+Co(50\%) & 599,8 & muy ab & 43,95 & media \\
\hline Gr1(II) & 778,4 & muy ab & 48,00 & media \\
\hline $\begin{array}{l}\text { Gr1(II)(50\%)+ } \\
\text { Gr2(III)(50\%) }\end{array}$ & 589,0 & muy ab & 23,50 & $\begin{array}{l}\text { extrem } \\
\text { bajo }\end{array}$ \\
\hline Bf $(80 \%)+M(20 \%)$ & 59,8 & no muy ab & \\
\hline
\end{tabular}

d)

\begin{tabular}{|l|c|l|l|l|}
\hline Material & Abr, g/ton & E. Cualit. & CLI & E. Cualit. \\
\hline Pf & 879,7 & muy ab & & fuera de rango \\
\hline Co & 319,9 & Ab media & & fuera de rango \\
\hline $\begin{array}{l}\text { Gr1(II)(50\%)+ } \\
\text { Co(50\%) }\end{array}$ & 549,2 & muy ab & & fuera de rango \\
\hline Pf(50\%)+Co(50\%) & 599,8 & muy ab & & fuera de rango \\
\hline Gr1(II) & 778,4 & muy ab & 15 & alta \\
\hline $\begin{array}{l}\text { Gr1(II)(50\%)+ } \\
\text { Gr2(III)(50\%) }\end{array}$ & 589,0 & muy ab & fuera de rango \\
\hline Bf (80\%)+M(20\%) & 59,8 & no muy ab \\
\hline
\end{tabular}

e)

\begin{tabular}{|l|c|l|c|l|}
\hline Material & Abr, g/ton & E. Cualit. & $\begin{array}{c}\text { I. Schi., } \\
\text { kN/m }\end{array}$ & E. Cualit. \\
\hline Pf & 879,7 & muy ab & 6,76 & muy alta \\
\hline Co & 319,9 & ab media & 0,26 & muy baja \\
\hline $\begin{array}{l}\text { Gr1(II)(50\%)+ } \\
\text { Co(50\%) }\end{array}$ & 549,2 & muy ab & 2,63 & media \\
\hline Pf(50\%)+Co(50\%) & 599,8 & muy ab & 3,51 & alta \\
\hline Gr1(II) & 778,4 & muy ab & 5,00 & alta \\
\hline $\begin{array}{l}\text { Gr1(II)(50\%)+ } \\
\text { Gr2(III)(50\%) }\end{array}$ & 589,0 & muy ab & 5,88 & muy alta \\
\hline Bf (80\%)+M(20\%) & 59,8 & no muy ab & \\
\hline
\end{tabular}

f)

\begin{tabular}{|l|c|l|l|c|}
\hline Material & Abr, g/ton & E. Cualit. & $\begin{array}{l}\text { RAI, } \\
\text { RCSxCEQ }\end{array}$ & $\begin{array}{l}\text { E. } \\
\text { Cualit. }\end{array}$ \\
\hline Pf & 879,7 & muy ab & 30,50 & abrasivo \\
\hline Co & 319,9 & ab media & 13,00 & baja \\
\hline $\begin{array}{l}\text { Gr1(II)(50\%)+ } \\
\text { Co(50\%) }\end{array}$ & 549,2 & muy ab & 29,93 & baja \\
\hline Pf(50\%)+Co(50\%) & 599,8 & muy ab & 21,19 & baja \\
\hline Gr1(II) & 778,4 & muy ab & 49,60 & abrasivo \\
\hline $\begin{array}{l}\text { Gr1(II)(50\%)+ } \\
\text { Gr2(III)(50\%) }\end{array}$ & 589,0 & muy ab & 40,30 & abrasivo \\
\hline \begin{tabular}{l} 
Bf( $80 \%)+M(20 \%)$ \\
\hline
\end{tabular} & 59,8 & no muy ab & \\
\hline
\end{tabular}




\section{Conclusiones}

De la aplicación de las fórmulas empíricas se desprende que no todos los procedimientos descritos en la literatura son de aplicación en cualquier tipo de roca o suelo sino que es muy importante que el tipo de roca o suelo a excavar tenga características similares a los materiales usados en la base de datos de referencia.

Por otra parte se ha de considerar la etapa en la que se encuentra el desarrollo del proyecto, ya que algunas fórmulas son apropiadas para una fase preliminar en la que se necesita sólo una idea global y general de los rendimientos de la excavación y, en cambio, otras son apropiadas para etapas más avanzadas. En otros casos puede ser necesario recurrir a métodos que permiten hacer un retro análisis y contrastar los valores predichos con valores reales para detectar posibles problemas y corregir a tiempo.

Finalmente es de destacar que hay aspectos del fenómeno que aún no han sido tenidos en cuenta ni incorporados a ninguna formulación como son, por ejemplo, el comportamiento estructural de la cabeza de corte cuya deformación no es uniforme $\mathrm{y}$, en consecuencia, no distribuye el esfuerzo a todos sus discos por igual sino que hay discos que están más presionados que otros. La rueda de corte experimenta deformaciones por temperatura y redistribución de esfuerzos que tiene que ver con la ubicación de los apoyos y su movimiento telescópico que acaban influyendo en el proceso de corte y desgaste de unos cortadores antes que en otros.

Otro aspecto que no es considerado por ningún modelo de predicción es la apertura de la cabeza de corte, que controla la velocidad de evacuación del material triturado desde dentro de la cámara. Si el material excavado es muy abrasivo el tiempo de permanencia de este tipo de material en la rueda incrementa el efecto de desgaste sobre las piezas de la rueda.

En cuanto a la abrasividad, se ha presentado un ensayo de laboratorio que es apropiado para terrenos con presencia de suelos mixtos y rocas blandas. El ensayo es relativamente simple y económico, dando resultados cualitativamente semejantes a los de otras medidas de abrasividad más comunes pero con menor rango de aplicación. En el futuro, y a partir de los datos de Can Zam y otros tramos de L9, se espera cuantificar la relación de esta medida con el desgaste o de consumo de cortadores observado durante la excavación.

\section{Referencias}

AFNOR (1990). Granulats - essai d'abrasivité et de broyabilité. Norme NF P18 - 579.

Bilgin, N. and Balci, C. (2005). Performance prediction of mechanical excavators in tunnels. Estambul, ITA/AITES.

Bieniawski, Z.T. (1989). Engineering rock mass classifications. John Wiley and Sons Inc.

Bruland, A. (1998). Hard rock tunnel boring. PhD thesis Norwegian University of Sciences and Technology of Trondheim.

Büchi, E., Mathier, J.F. and Wyss, Ch. (1995). Rock abrasivity - a significant cost factor for mechanical tunnelling in loose and hard rock. Tunnel 5, 38 - 43.

Burger, W. (2006). Hard rock cutterhead design. Proceedings of the North American Tunnelling Conference, 257-263.

Casinelli, F., Cina, S., Innaurato, N. and Mancini, R. (1982). Power consumption and metal wear in tunnel boring machines: Analysis of tunnel boring operations in hard rock. London, IMM, 73-81.

Cerchar (1986). The Cerchar Abrasiveness Index. Centre d' Etudes et Recherches de Charbonnages de France, Verneuil.

Eusebio, A., Grasso, P., Mahtab, A. and Innaurato, A. (1991). Rock characterization for selection of a TBM for a railway tunnel near Geneva, Italy. Proceedings Int. Symp. on Mine Mech. and Automation, Colorado, CSM/USBM,Vol. 1, 4-25 to 4-35.

Farmer, I.W. and Glossop, N.H. (1980). Mechanics of disc cutter penetration. Tunnels and Tunnelling 12(6), 22-25.

Festl, J. (2006). The LCPC test - A possibility to determine soil abrasivity? BA thesis Technische Universität München (in German).

Frenzel, C., Käsling, H. and Thuro, K. (2008). Factors influencing disc cutter wear. Geomechanik und Tunnelbau 1(1), 55 - 60.

Gehring, K. (1995). Leistungs - und Verschleibprognosen im maschinellen Tunnelbau. Felsbau 13(6), 439-448.

Graham, P.C. (1976). Rock exploration for machine manufacturers. Proceedings of the Symposium on Exploration for Rock Engineering, vol. 1, Johannesburg, Balkema, 173-180. 
Gutiérrez Manjón, J.M. (2009). Modo de operación y consumo de cortadores en los terrenos metamórficos y graníticos de los túneles de Guadarrama (AVE), Línea 9 Metro de Barcelona, Vigo (AVE). Barcelona, UPC.

Hughes, H.M. (1986). The relative cuttability of coal measures rock. Mining Science and Technology 3(2), 95-109.

Kahraman, S., Bilgin, N. and Feridunoglu, C. (2003). Dominant rock propierties affecting the penetration rate of percusive drills. International Journal of Rock Mechanics and Mining Sciences 40(5), 711-723.

Käsling, H. and Thuro, K. (2010). Determining abrasivity of rock and soil in the laboratory. In Williams et al. (eds.) Geologically Active. Proceedings of the $11^{\text {th }}$ Congress of the International Association for Engineering Geology and the Environment, Auckland, New Zealand, Taylor \& Francis, London.

Lien, R. (1961). An indirect test method for estimating the drillability of rocks. Dr. thesis Trondheim University.

Movinkel, T. and Johannessen, O. (1986). Geological parameters for hard rock tunnel boring. Tunnels \& Tunnelling 4, 45 - 48.

Nielsen, B., Dahl, F. and Holzhäuser, J. (2006a). Abrasivity testing for rock and soils. Tunnels \& Tunnelling International, $47-49$.
Nielsen, B., Dahl, F. and Holzhäuser, J. (2006b). Abrasivity of soils in TBM tunnelling. Tunnels \& Tunnelling International, 36 - 38.

Ozdemir, L. (2003). CSM computer model for TBM performance predictions. Colorado School of Mines.

Ozdemir, L. and Wang, F. (1979). Mechanical tunnel boring, prediction and machine design. Finat Report NSF/RA-790161: 204.

Plinninger, R. J. and Restner, U. (2008). Abrasiveness testing, Quo Vadis? - A commented overview of abrasiveness testing methods. Geomechanik und Tunnelbau 1(1), 61-70.

Roxbosough, F.F. and Phillips, H.R. (1975). Rock excavation by disc cutter. International Journal of Rock Mechanics and Mining Sciences 12(12), 361-366.

Sapigni, M., Berti, M., Bethaz, E., Busillod, A. and Cardone, G. (2002). TBM performance estimation using rock mass classifications. International Journal of Rock Mechanics and Mining Sciences 39(6), 771-788.

Thuro, K., Singer, J., Käsling, H. and Bauer, M. (2006). Soil abrasivity assessment using the LCPC testing device. Felsbau 24(6), 37 - 45 . 